Tôhoku Math. Journ.

39 (1987), 557-574.

\title{
GLOBAL CONVERGENCE OF SUCCESSIVE APPROXIMATIONS OF SOLUTIONS FOR FUNCTIONAL DIFFERENTIAL EQUATIONS WITH INFINITE DELAY
}

\author{
JoNG SoN SHIN \\ (Received September 16, 1986)
}

1. Introduction. Let $E$ be a Banach space with norm $|\cdot|_{E}$ and let $J=[\sigma, \sigma+a), 0<a \leqq \infty$. If $x:(-\infty, \sigma+a) \rightarrow E$, then for any $t \in$ $(-\infty, \sigma+a)$ we define $x_{t}:(-\infty, 0] \rightarrow E$ by $x_{t}(\theta)=x(t+\theta),-\infty<\theta \leqq 0$. In this paper we consider the initial value problem for functional differential equations with infinite delay (IP);

$$
\begin{aligned}
& \frac{d x}{d t}=f\left(t, x_{t}\right), \quad t>\sigma \\
& x_{\sigma}=\varnothing \in \mathscr{B},
\end{aligned}
$$

where $f$ is an $E$-valued mapping defined on $J \times \mathscr{B}$ and $\mathscr{B}$ is an abstract phase space with semi-norm or quasi-norm $|\cdot|_{\varnothing}$ satisfying suitable axioms introduced by Hale and Kato [2].

The purpose of this paper is to give sufficient conditions for the global convergence of successive approximations for (IP): main results are Theorems 4.1, 4.2, 4.3 and 4.4, which extend results obtained in [1], [6], [7], [11]. Needless to say, our results ensure the global existence of a unique solution to (IP).

Recently, the author [9] has proved the uniqueness and the global convergence of successive approximations of solutions for functional integral equations under some Perron-type conditions. For (1.1) these conditions become

$$
|f(t, \varphi)-f(t, \psi)| \leqq \omega_{1}\left(t,|\varphi-\psi|_{\mathscr{\infty}}\right)
$$

and

$$
\left|f\left(t, x_{t}\right)-f\left(t, y_{t}\right)\right| \leqq \omega_{2}\left(t, \sup _{o \leq s \leq t}|x(s)-y(s)|\right),
$$

where real-valued functions $\omega_{i}, i=1,2$, are integrable and satisfy some uniqueness conditions. On the other hand, the author [10] has proved Kamke's uniqueness theorems of solutions for (IP). In this paper we shall show the global convergence of successive approximations for (IP) 
under Kamke's uniqueness conditions with (1.3) or (1.4), provided $\omega_{i}(t, s)$ is nondecreasing in $s$. Theorems $4.1,4.2$ and 4.3 are related to the inequality (1.3) and Theorem 4.4 is concerned with (1.4). In general, Theorem 4.3 is a generalization of Theorems 4.1 and 4.2 under the additional axioms on the phase space. Theorem 4.2 depends on a quantity $\beta_{\nu}$ defined by

$$
\beta_{\nu}=\limsup _{h \rightarrow 0+} \frac{1}{h}\{\|S(h)\|-1\}
$$

(for the definition of $\|S(h)\|$ see Section 2 below). Theorem 4.4 is essentially a corollary to Theorem 4.1 (cf. [9]). However, both Theorems 4.3 and 4.4 are useful in showing the convergence of the successive approximations of solutions for (IP). Such examples will be given in Section 6. When the delay is finite, that is, $\mathscr{B}=C([-r, 0], E), 0 \leqq r<\infty$, Theorems 4.1, 4.2 and 4.3 are equivalent to one another and Theorem 4.3 is contained in Theorem 4.4. Finally, we note that our results apply to linear systems, though the results obtained in [7], [11] do not.

2. Phase space $\mathscr{B}$ and assumptions. Let $R^{-}=(-\infty, 0], R^{+}=$ $[0, \infty), R=(-\infty, \infty)$ and let $\mathscr{B}=\mathscr{B}\left(R^{-}, X\right)$ be a linear space of functions mapping $R^{-}$into $X$ with semi-norm or quasi-norm $|\cdot|_{\infty}$, where $X=E$ or $R$. If we wish to emphasize the dependence on the space $X$, we write $\mathscr{B}^{X}$. So far as there is no fear of confusion, we denote various kinds of norms and semi-norms (or quasi-norms) by the same symbol $|\cdot|$. For $\varphi$ and $\psi$ in $\mathscr{B}, \varphi=\psi$ means that $\varphi(\theta)=\psi(\theta)$ for all $\theta \in R^{-}$. For $\varphi \in \mathscr{B}$, let $X_{\sigma}^{\varphi}[\delta]$ (or $\left.X_{\sigma}^{\varphi}(\delta)\right)$ be the set of all functions $x$ mapping $(-\infty$, $\sigma+\delta$ ] (or $(-\infty, \sigma+\delta)), \delta>0$, to $X$ such that $x_{o}=\phi$ and $x(t)$ is continuous on $[\sigma, \sigma+\delta]$ (or $[\sigma, \sigma+\delta))$. Throughout this paper we assume that the following axioms on the phase space $\mathscr{B}$ are always satisfied:

$\left(\mathrm{B}_{1}\right)$ If $x \in X_{\sigma}^{\varphi}(A)$, then $x_{t}$ also lies in $\mathscr{B}$ for all $t \in[\sigma, \sigma+A)$ and $x_{t}$ is continuous in $t$ on $[\sigma, \sigma+A)$.

$\left(\mathrm{B}_{2}\right)$ There exist functions $K(t)>0$ and $M(t) \geqq 0$ with the following properties:

(i) $K(t)$ is continuous for $t$ in $R^{+}$.

(ii) $M(t)$ is locally bounded on $R^{+}$.

(iii) For every function $x \in X_{o}^{\varphi}(A)$, it holds that

$$
\left|x_{t}\right| \leqq K(t-\sigma) \sup \{|x(s)|: \sigma \leqq s \leqq t\}+M(t-\sigma)\left|x_{\sigma}\right| \quad \text { for } t \in[\sigma, \sigma+A) \text {. }
$$

$\left(\mathrm{B}_{3}\right) \quad$ There is a positive constant $L$ such that $|\varphi(0)| \leqq L|\varphi|_{\mathscr{\infty}}$ for all $\varphi \in \mathscr{B}$. 
Two examples of the phase space $\mathscr{B}$ below were given in [2].

EXAmple 2.1. Let $\gamma \in R$. The space $\mathscr{C}_{r}^{E}$ is the space of continuous functions $\varphi$ mapping $R^{-}$into $E$ having the limit $\lim _{s \rightarrow-\infty} e^{r s} \varphi(s)$ with the norm $|\varphi|_{\mathscr{C}_{r}^{E}}=\sup _{\boldsymbol{s} \in R^{-}} e^{\gamma s}|\varphi(s)|_{E}$.

EXAMPLE 2.2. Let

$\mathscr{L}^{E}=\left\{\varphi: R^{-} \rightarrow E:\right.$ measurable on $(-\infty,-r]$, continuous on

$$
\left.[-r, 0] \text { and }|\varphi|_{E}<\infty\right\} \text {, }
$$

where $0 \leqq r<\infty$ and

$$
|\varphi|_{\mathscr{E} E}=\sup _{-r \leq s \leq 0}|\varphi(s)|_{E}+\int_{-\infty}^{0} e^{\mathbf{s}}|\varphi(s)|_{E} d s .
$$

For the other examples of the phase space $\mathscr{B}$, refer to [8], [9]. Hereafter, let $K_{\delta}=\sup \{K(s): 0 \leqq s \leqq \delta\}$ and $X_{\sigma}^{\varphi}[\delta, \gamma]=\left\{x \in X_{\sigma}^{\varphi}[\delta]: \sup \{\mid x(s)-\right.$ $x(\sigma) \mid: \sigma \leqq s \leqq \sigma+\delta\} \leqq \gamma\}$.

Next, we assume that $f$ in (IP) is a mapping from $J \times \mathscr{B}$ into $E$ satisfying the following conditions:

$\left(\mathrm{F}_{1}\right) f(\cdot, \varphi)$ is strongly measurable in $t \in J$ for each fixed $\varphi \in \mathscr{B}$.

$\left(\mathrm{F}_{2}\right) f(t, \cdot)$ is continuous in $\varphi \in \mathscr{B}$ for a.a. $t \in J$.

$\left(\mathrm{F}_{8}\right)$ For every closed bounded set $B \subset \mathscr{B}$ there exists a locally integrable function $\beta_{B}(t)$, defined on $J$, such that $|f(t, \psi)| \leqq \beta_{B}(t)$ for a.a. $t \in J$ and all $\psi \in \mathscr{B}$.

In order to give conditions for the convergence of successive approximations for (IP), we introduce a Kamke-type function $\omega$ as follows. A function $\omega$ is said to be a Kamke-type function with domain $\left(t_{0}, t_{0}+d\right] \times[0,2 r]$ if the following conditions hold:

$\left(\omega_{1}\right) \quad \omega=\omega(t, s)$ is a real-valued function, defined on $\left(t_{0}, t_{0}+d\right] \times[0,2 r]$, which is Lebesgue measurable in $t$ for each fixed $s \in[0,2 r]$ and is continuous and nondecreasing in $s$ for a.a. $t \in\left[t_{0}, t_{0}+d\right]$.

$\left(\omega_{2}\right)$ There exists a function $\alpha$, defined on $\left(t_{0}, t_{0}+d\right]$ and locally integrable there, such that $|\omega(t, s)| \leqq \alpha(t)$ for a.a. $t \in\left(t_{0}, t_{0}+d\right]$ and all $s \in[0,2 r]$.

In case that the function $\alpha$ arising in $\left(\omega_{2}\right)$ is integrable over $\left(t_{0}, t_{0}+d\right]$, we say that $\omega$ is a Perron-type function.

Now, we present some conditions on a Kamke (or Perron)-type function $\omega$ with domain $\left(t_{0}, t_{0}+d\right] \times[0,2 r]$, which are essential in this paper.

$\left(\Omega_{1}\right) \quad z \equiv 0$ is the unique absolutely continuous function which satisfies the initial condition $z\left(t_{0}\right)=0$ and

$$
\frac{d z}{d t}=\omega\left(t, K\left(t-t_{0}\right) z(t)\right) \quad \text { for a.a. } t \in\left(t_{0}, t_{0}+d\right] .
$$


$\left(\Omega_{2}\right) \quad z \equiv 0$ is the unique absolutely continuous function which satisfies the initial condition $z\left(t_{0}\right)=0$ and

$$
\frac{d z}{d t}=\omega(t, z(t)) \quad \text { for a.a. } t \in\left(t_{0}, t_{0}+d\right] .
$$

Define linear operators $S(t): \mathscr{B} \rightarrow \mathscr{B}, t \geqq 0$, by

$$
[S(t) \varphi](\theta)= \begin{cases}\varphi(t+\theta) & \text { if } t+\theta \leqq 0 \\ \varphi(0) & \text { if } t+\theta>0 .\end{cases}
$$

Then we define a quantity $\beta_{\nu}$ by the relation

$$
\beta_{\nu}=\limsup _{h \rightarrow 0+} \frac{1}{h}\{\|S(h)\|-1\},
$$

where $-\infty \leqq \beta_{\nu} \leqq \infty$ and $\|S(t)\|=\sup \left\{|S(t) \varphi|_{\mathscr{S}}:|\varphi|_{\mathscr{\varnothing}} \leqq 1\right\}$. Throughout this paper we assume that $\beta_{\nu} \in R$ (cf. [10]).

$\left(\Omega_{3}\right) \quad z \equiv 0$ is the unique absolutely continuous function which satisfies the initial condition $z\left(t_{0}\right)=0$ and

$$
\frac{d z}{d t}=K(0) \omega(t, z(t))+\beta_{\iota} z(t) \quad \text { for a.a. } t \in\left(t_{0}, t_{0}+d\right] .
$$

$\left(\Omega_{4}\right) \quad z \equiv 0$ is the unique absolutely continuous function mapping $\left(-\infty, t_{0}+d\right]$ into $R$, which satisfies the initial condition $z_{t_{0}}=0 \in \mathscr{B}^{R}$ and

$$
\left|\frac{d z}{d t}\right| \leqq \omega\left(t,\left|z_{t}\right|_{\mathscr{B} R}\right) \quad \text { for a.a. } \quad t \in\left(t_{0}, t_{0}+d\right] .
$$

$\left(\Omega_{5}\right) \quad z \equiv 0$ is the unique absolutely continuous function mapping $\left(-\infty, t_{0}+d\right]$ into $R$, which satisfies the initial condition $z_{t_{0}}=0 \in \mathscr{B}^{R}$ and

$$
\frac{d z}{d t}=\omega\left(t,\left|z_{t}\right|_{\mathscr{B} R}\right) \quad \text { for a.a. } \quad t \in\left(t_{0}, t_{0}+d\right] .
$$

Furthermore, we say that the function $\omega$ satisfies the condition $\left(\Omega_{i}\right)$, $i=1, \cdots, 5$, with $\left(D^{+} z\right)\left(t_{0}\right)=0$ if the condition $\left(D^{+} z\right)\left(t_{0}\right)=0$ is added to the initial condition in $\left(\Omega_{i}\right)$, where $\left(D^{+} z\right)\left(t_{0}\right)=\lim _{t \rightarrow t_{0}+0} z(t) /\left(t-t_{0}\right)$. Here we note that the condition $\left(\Omega_{i}\right)$ is slightly stronger than the condition $\left(\Omega_{i}\right)$ with $\left(D^{+} z\right)\left(t_{0}\right)=0$. The relationship among the conditions $\left(\Omega_{i}\right)$, $i=1, \cdots, 5$, are given in Section 3 .

REMark 2.1. If $\omega$ is a Perron-type function with domain $\left(t_{0}, t_{0}+d\right] \times$ $[0,2 r]$, then $(2.1)$ in the condition $\left(\Omega_{1}\right)$ can be replaced by a Volterra integral equation 
(cf. [9]).

$$
z(t)=K\left(t-t_{0}\right) \int_{t_{0}}^{t} \omega(s, z(s)) d s
$$

REMARK 2.2. If the function $K(t)$ in $\left(\mathrm{B}_{2}\right)$ is continuously differentiable, then (2.1) in the condition $\left(\Omega_{1}\right)$ is equivalent to the equation $d z / d t=$ $K^{\prime}(t) z(t) / K(t)+K(t) \omega(t, z(t))$, where the prime denotes the differentiation with respect to $t$. Indeed, if we set $w(t)=K(t) z(t)$, where $z(t)$ is a solution of (2.1), then $w(t)$ is a solution of the above equation. In particular, if $K(t) \equiv K$ in $\left(\mathrm{B}_{2}\right)$, then (2.1) is reduced to the equation $d z / d t=K \omega(t, z(t))$.

Definition 2.1. Suppose that $\left(\mathrm{F}_{1}\right)-\left(\mathrm{F}_{3}\right)$ are satisfied. Then a function $u:(-\infty, \sigma+b] \rightarrow E$ is said to be a solution of (IP) on $[\sigma, \sigma+b]$ if $u$ is an absolutely continuous function on $[\sigma, \sigma+b]$ with a strong derivative $d u / d t$ satisfying (1.1) and (1.2) for a.a. $t \in[\sigma, \sigma+b]$.

DEFINITION 2.2. Let $f$ be a continuous mapping from $J \times \mathscr{B}$ into $E$. If a function $u:(-\infty, \sigma+b] \rightarrow E$ satisfies (1.1) and (1.2) for all $t \in$ $[\sigma, \sigma+b]$, then it is said to be a $C^{1}$-solution of (IP) on $[\sigma, \sigma+b]$.

3. Comparison results. In this section we present several comparison results related with the axioms on the phase space in order to show the convergence of successive approximations for solutions of (1.1). Further we state relationship among the conditions $\left(\Omega_{i}\right)$ or $\left(\Omega_{i}\right)$ with $\left(D^{+} z\right)\left(t_{0}\right)=0$, $i=1, \cdots, 5$. Let $\bar{D}_{+}$and $D_{+}$denote the right hand upper derivative and the right hand derivative, respectively. Let $C(I, E)$ be the space of continuous functions mapping $I, I \subset R$, into $E$ with the supremum norm.

LEMmA 3.1. Suppose $\mathscr{B}$ satisfies the axioms $\left(\mathrm{B}_{1}\right)$ and $\left(\mathrm{B}_{2}\right)$ and let $x$ belong to $X_{\sigma}^{\varphi}(A)$. Then it holds that for any fixed $t \in[\sigma, \sigma+A)$,

$$
\bar{D}_{+}\left|x_{t}\right|_{\mathscr{B}} \leqq K(0)\left|D_{+} x(t)\right|+\beta_{\nu}\left|x_{t}\right|_{\mathscr{B}},
$$

whenever $D_{+} x(t)$ exists. In particular, in case $\mathscr{B}=C([-r, 0], E), 0 \leqq$ $r<\infty,(3.1)$ is reduced to

$$
\bar{D}_{+}\left|x_{t}\right|_{\mathscr{G}} \leqq\left|D_{+} x(t)\right| \text {. }
$$

LEMMA 3.2. Let $x(t)$ be an absolutely continuous function from $[a, b]$ into $E$. Then the following inequality holds:

$$
\frac{d}{d t}\left(\sup _{a \leqq s \leqq t}|x(s)|\right) \leqq\left|\frac{d}{d t}\right| x(t)|| \leqq\left|\frac{d x(t)}{d t}\right| \quad \text { for a.a. } t \in(a, b) .
$$

For a proof of Lemmas 3.1 and 3.2 see [10, Lemmas 3.1 and 3.2].

LEMMA 3.3. Let $\omega$ be a Kamke-type function with domain $\left(t_{0}, t_{0}+d\right] \times$ 
$[0,2 r]$. Assume that the condition $\left(\Omega_{2}\right)$ with $\left(D^{+} z\right)\left(t_{0}\right)=0$ is satisfied. If $u:\left[t_{0}, t_{0}+d\right] \rightarrow[0,2 r]$ is an absolutely continuous function satisfying the condition $u\left(t_{0}\right)=\left(D^{+} u\right)\left(t_{0}\right)=0$ and the differential inequality

$$
\frac{d u(t)}{d t} \leqq \omega(t, u(t)) \quad \text { for a.a. } t \in\left(t_{0}, t_{0}+d\right],
$$

then $u(t) \equiv 0$ on $\left[t_{0}, t_{0}+d\right]$.

For a proof of this lemma see [10, Lemma 4.1].

LEMMA 3.4. Let $\omega$ be a Kamke-type function with domain $\left(t_{0}, t_{0}+d\right] \times$ $[0,2 r]$. Assume that the condition $\left(\Omega_{1}\right)$ with $\left(D^{+} z\right)\left(t_{0}\right)=0$ is satisfied. If $u:\left[t_{0}, t_{0}+d\right] \rightarrow\left[-2 r / K_{d}, 2 r / K_{d}\right]$ is an absolutely continuous function satisfying the condition $u\left(t_{0}\right)=\left(D^{+} u\right)\left(t_{0}\right)=0$ and the differential inequality

$$
\left|\frac{d u}{d t}\right| \leqq \omega\left(t, K\left(t-t_{0}\right) \sup _{t_{0} \leqq s \leqq t}|u(s)|\right) \quad \text { for a.a. } t \in\left(t_{0}, t_{0}+d\right],
$$

then $u(t) \equiv 0$ on $\left[t_{0}, t_{0}+d\right]$.

Proof. Set $\tilde{u}(t)=\sup _{t_{0} \leqq s \leqq t}|u(s)|$. Then by Lemma 3.2, (3.4) is reduced to

$$
\frac{d \tilde{u}}{d t} \leqq \omega\left(t, K\left(t-t_{0}\right) \tilde{u}(t)\right) \quad \text { for a.a. } t \in\left(t_{0}, t_{0}+d\right] .
$$

Thus it follows from Lemma 3.3 that $\tilde{u}(t) \equiv 0$, and hence $u(t) \equiv 0$. q.e.d.

LEMmA 3.5. Suppose $\mathscr{B}^{R}$ satisfies the axioms $\left(\mathrm{B}_{1}\right)-\left(\mathrm{B}_{3}\right)$ and let $\omega$ be a Kamke-type function with domain $\left(t_{0}, t_{0}+d\right] \times[0,2 r]$. Assume that either the condition $\left(\Omega_{1}\right)$ with $\left(D^{+} z\right)\left(t_{0}\right)=0$ or the condition $\left(\Omega_{3}\right)$ with $\left(D^{+} z\right)\left(t_{0}\right)=0$ is satisfied. If $w:\left(-\infty, t_{0}+d\right] \rightarrow\left[-2 r / K_{d}, 2 r / K_{d}\right]$ is an absolutely continuous function satisfying the initial conditions $w_{t_{0}}=$ $0 \in \mathscr{B}^{R}$ and $\left(D^{+} w\right)\left(t_{0}\right)=0$ and (2.6), then $w(t) \equiv 0$ on $\left[t_{0}, t_{0}+d\right]$.

Proof. Assume that the condition $\left(\Omega_{1}\right)$ with $\left(D^{+} z\right)\left(t_{0}\right)=0$ is satisfied. Then, by the axiom $\left(\mathrm{B}_{2}\right)$ and the property that $\omega(t, s)$ is nondecreasing in $s$, we have

$$
\left|\frac{d w}{d t}\right| \leqq \omega\left(t,\left|w_{t}\right|_{S B R}\right) \leqq \omega\left(t, K\left(t-t_{0}\right) \sup _{t_{0} \leqq s \leqq t}|w(s)|\right) .
$$

By Lemma 3.4, we see that $w(t) \equiv 0$.

Next, Assume that the condition $\left(\Omega_{3}\right)$ with $\left(D^{+} z\right)\left(t_{0}\right)=0$ is satisfied. By Lemma 3.1, we have

$$
\bar{D}_{+}\left|w_{t}\right|_{\mathscr{B}} R \leqq K(0) \omega\left(t,\left|w_{t}\right|_{\mathscr{B}}\right)+\beta_{\nu}\left|w_{t}\right|_{\overparen{B}} \quad \text { for a.a. } t \in\left(t_{0}, t_{0}+d\right] .
$$

Thus, setting $z(t)=\left|w_{t}\right|_{\circledast}$, we reduce the above inequality to 


$$
\frac{d z(t)}{d t} \leqq K(0) \omega(t, z(t))+\beta_{\nu} z(t) \quad \text { for a.a. } t \in\left(t_{0}, t_{0}+d\right],
$$

because $z(t)$ is absolutely continuous on $\left[t_{0}, t_{0}+d\right]$ (see [10, Lemma 3.3]). It is easy to show that $\left(D^{+} z\right)\left(t_{0}\right)=0$. Therefore we can obtain $z(t) \equiv 0$ by Lemma 3.3, which implies that $w(t) \equiv 0$ by $\left(\mathrm{B}_{3}\right)$.

q.e.d.

In order to state the following lemma, in addition to $\left(\mathrm{B}_{1}\right)-\left(\mathrm{B}_{3}\right)$, we must impose the following axiom $\left(\mathrm{B}_{4}\right)$ on the phase space $\mathscr{B}^{R}$ :

$\left(\mathrm{B}_{4}\right)$ If $\phi$ and $\psi$ are in $\mathscr{B}^{R}$ and $|\varphi(\theta)| \leqq|\psi(\theta)|$ for a.a. $\theta \in R^{-}$, then the inequality $|\varphi|_{\mathscr{B}} R \leqq|\psi|_{\mathscr{B}} R$ holds.

LEMMA 3.6. Suppose the phase space $\mathscr{B}^{R}$ satisfies the axioms $\left(\mathrm{B}_{1}\right)-$ $\left(\mathrm{B}_{4}\right)$ and let $\omega$ be a nonnegative and Perron-type function with domain $\left(t_{0}, t_{0}+d\right] \times[0,2 r]$. Assume further that the condition $\left(\Omega_{\mathrm{b}}\right)$ is satisfied. If $m:\left(-\infty, t_{0}+d\right] \rightarrow R^{+}$is an absolutely continuous function satisfying the initial condition $m_{t_{0}}=0 \in \mathscr{B}^{R}$ and the differential inequality

$$
\frac{d m}{d t} \leqq \omega\left(t,\left|m_{t}\right|_{\mathscr{B}} R\right) \quad \text { for a.a. } t \in\left(t_{0}, t_{0}+d\right] \text {, }
$$

then $m(t) \equiv 0$ on $\left[t_{0}, t_{0}+d\right]$,

Proof. Let $\varepsilon^{n}: R^{-} \rightarrow R^{+}, n=1,2, \cdots$, be continuous and nondecreasing. Suppose that

(1) $\operatorname{supp} \varepsilon^{n} \subset[-1,0]$ for all $n$;

(2) $\left|\varepsilon^{n}\right|_{[-1,0]} \rightarrow 0$ as $n \rightarrow \infty$, where $\left|\varepsilon^{n}\right|_{[-1,0]}=\sup _{-1 \leq \theta \leq 0}\left|\varepsilon^{n}(\theta)\right|$;

(3) $\varepsilon^{n+1}(\theta) \leqq \varepsilon^{n}(\theta)$ for each $\theta \in(-\infty, 0)$ and for each $n$;

(4) $0<\varepsilon^{n+1}(0)<\varepsilon^{n}(0)$ for each $n$.

Denote by $u^{n}(t)$ any solution of the scalar differential equation

$$
\frac{d u}{d t}=\omega\left(t,\left|u_{t}\right|_{s}\right), \quad u_{t_{0}}=\varepsilon^{n} \in \mathscr{B}^{R} .
$$

Then, it is not difficult to show that $0 \leqq u^{n+1}(t) \leqq u^{n}(t)$ on $\left[t_{0}, t_{0}+d\right]$ for a sufficiently large $n$ and that $\left|u^{n}\right|_{\left[t_{0}, t_{0}+d\right]} \rightarrow 0$ as $n \rightarrow \infty$. To complete the proof, it is enough to prove that $m(t) \leqq u^{n}(t)$ for $t \in\left[t_{0}, t_{0}+d\right]$. Suppose, for a contradiction, that $\mathscr{Z}=\left\{t \in\left[t_{0}, t_{0}+d\right]: m(t)>u^{n}(t)\right\}$ is nonempty, and let $t_{1}=\inf \mathscr{Z}$. Since $m\left(t_{0}\right)<\varepsilon^{n}(0)=u^{n}\left(t_{0}\right)$, we have $t_{1}>t_{0}, m\left(t_{1}\right)=u^{n}\left(t_{1}\right)$ and $m(t) \leqq u^{n}(t)$ for $t \in\left[t_{0}, t_{1}\right]$. Thus we have $0 \leqq$ $m(t+\theta) \leqq u^{n}(t+\theta)$ for $t \in\left[t_{0}, t_{1}\right]$ and $\theta \in R^{-}$, which implies that $\left|m_{t}\right|_{\mathscr{B}} R \leqq$ $\left|u_{t}^{n}\right|_{\mathscr{G}} R$ for $t \in\left[t_{0}, t_{1}\right]$ by the axiom $\left(\mathrm{B}_{4}\right)$. Since $m(t)$ is absolutely continuous on $\left[t_{0}, t_{0}+d\right], d m(t) / d t$ exists for a.a. $t \in\left[t_{0}, t_{0}+d\right]$ and is an integrable function. Thus, by the property that $\omega(t, s)$ is nondecreasing in $s$, we have 


$$
0=u^{n}\left(t_{1}\right)-m\left(t_{1}\right)=\varepsilon^{n}(0)+\int_{t_{0}}^{t_{1}}\left[\omega\left(s,\left|u_{s}^{n}\right|_{\mathscr{B} R} R\right)-\omega\left(s,\left|m_{s}\right|_{\mathscr{B}} R\right)\right] d s \geqq \varepsilon^{n}(0)>0,
$$

which is a contradiction. Thus the set $\mathscr{Z}$ is empty.

q.e.d.

Finally, for special spaces we summarize relationship among the conditions $\left(\Omega_{i}\right)$ with $\left(D^{+} z\right)\left(t_{0}\right)=0, i=1, \cdots, 5$. Let $\omega$ be a Kamke-type function.

(1) In general, the condition $\left(\Omega_{1}\right)$ with $\left(D^{+} z\right)\left(t_{0}\right)=0$ or the condition $\left(\Omega_{3}\right)$ with $\left(D^{+} z\right)\left(t_{0}\right)=0$ is stronger than the condition $\left(\Omega_{4}\right)$ with $\left(D^{+} z\right)\left(t_{0}\right)=0$, because of Lemma 3.5. Though the condition $\left(\Omega_{5}\right)$ with $\left(D^{+} z\right)\left(t_{0}\right)=0$ is weaker than the condition $\left(\Omega_{4}\right)$ with $\left(D^{+} z\right)\left(t_{0}\right)=0$, the converse remains open. However if $\omega$ is a Perron-type function, the conditions $\left(\Omega_{4}\right)$ and $\left(\Omega_{5}\right)$ are equivalent to each other by Lemma 3.6.

(2) Consider the case $\mathscr{B}=C([-r, 0], R), 0 \leqq r<\infty$. It is clear that $K(t) \equiv 1$ in $\left(\mathrm{B}_{2}\right)$ and $\beta_{\nu}=0$ in (2.4) (see [10]). In this case, the conditions $\left(\Omega_{i}\right)$ with $\left(D^{+} z\right)\left(t_{0}\right)=0, i=1, \cdots, 5$, are equivalent to one another.

(3) Consider the case $\mathscr{B}=\mathscr{C}_{r}^{R}$. It is clear that $K(t)=\sup _{-t \leqq \theta \leqq 0} e^{\gamma \theta}$ in $\left(\mathrm{B}_{2}\right)$ and $\beta_{\nu}=-\gamma$ if $\gamma<0$ while $\beta_{\nu}=0$ if $\gamma \geqq 0$ in (2.4) (see [2], [10]). Thus if $\gamma \geqq 0$, then the condition $\left(\Omega_{i}\right)$ with $\left(D^{+} z\right)\left(t_{0}\right)=0, i=1, \cdots, 5$, are equivalent to one another, because $\left|z_{t}\right|_{\mathscr{B}}=z(t)$, provided $z(t)$ : $\left(-\infty, t_{0}+d\right] \rightarrow R^{+}$is nondecreasing in $t$ and $z_{t_{0}}=0 \in \mathscr{B}^{R}$.

4. Convergence of successive approximations. We define the successive approximations for (IP) as follows:

$$
y^{n}(t)= \begin{cases}\varphi(t-\sigma) & \text { for } t \in(-\infty, \sigma] \\ \varphi(0)+\int_{\sigma}^{t} f\left(s, y_{s}^{n-1}\right) d s & \text { for } t \in[\sigma, \sigma+a),\end{cases}
$$

for $n \geqq 1$, where $y^{0} \in X_{o}^{\varphi}(a)$.

Now, we get the main results in this paper.

THEOREM 4.1. Suppose the phase space $\mathscr{B}^{E}$ satisfies the axioms $\left(\mathrm{B}_{1}\right)-\left(\mathrm{B}_{3}\right)$ and suppose the conditions $\left(\mathrm{F}_{1}\right)-\left(\mathrm{F}_{3}\right)$ are satisfied. Then the successive approximations $\left\{y^{n}(t)\right\}$ defined above converge uniformly on every compact interval of $J$ to a unique solution of (IP) if the following conditions are satisfied:

(i) The successive approximations $\left\{y^{n}(t)\right\}$ defined on $J$ are uniformly bounded on $[\sigma, \gamma]$ for every $\gamma \in(\sigma, \sigma+a)$.

(ii) For each $t_{0} \in J$ and $\xi \in X_{o}^{\varphi}\left[t_{0}-\sigma\right]$, there exist positive constants $d, r$ and a Kamke-type function $\omega$ with domain $\left(t_{0}, t_{0}+d\right] \times[0,2 r]$ for which the condition $\left(\Omega_{1}\right)$ and the following condition holds: 
$\left(\mathrm{F}_{4}\right) \quad$ If $x, y \in X_{\sigma}^{\varphi}\left[t_{0}+d-\sigma\right], x_{t}, y_{t} \in S\left(\xi_{t_{0}}, r\right)$ and $|x-y|_{[\sigma, t]} \leqq 2 r$ for $t \in\left[t_{0}, t_{0}+d\right]$, then

$$
\left|f\left(t, x_{t}\right)-f\left(t, y_{t}\right)\right| \leqq \omega\left(t,\left|x_{t}-y_{t}\right|_{\mathscr{B}} R\right) \quad \text { for a.a. } t \in\left(t_{0}, t_{0}+d\right],
$$

where $S(\psi, r)$ denotes a closed ball with center at $\psi \in \mathscr{B}^{X}$ and radius $r$, and $|x|_{[\sigma, t]}=\sup _{\sigma \leqq s \leqq t}|x(s)|$.

THEOREM 4.2. The conclusion of Theorem 4.1 remains true if the condition $\left(\Omega_{1}\right)$ is replaced by the condition $\left(\Omega_{3}\right)$.

Before stating the following theorem, we observe that from the axiom $\left(\mathrm{B}_{1}\right)$ the phase space $\mathscr{B}^{E}$ contains all the continuous functions on $R^{-}$into $E$ with compact support.

THEOREM 4.3. Suppose the phase space $\mathscr{B}^{E}$ satisfies the axioms $\left(\mathrm{B}_{1}\right)-\left(\mathrm{B}_{3}\right)$ while the phase space $\mathscr{B}^{R}$ satisfies the axioms $\left(\mathrm{B}_{1}\right)-\left(\mathrm{B}_{4}\right)$. Suppose further that $\mathscr{B}^{E}$ and $\mathscr{B}^{R}$ are related to each other as follows:

(A) If $\varphi: R^{-} \rightarrow E$ is a continuous function with compact support, then the inequality $|\varphi|_{\mathscr{B} E} \leqq|\langle\varphi\rangle|_{\mathscr{B} R}$ holds, where the function $\langle\varphi\rangle: R^{-} \rightarrow R^{+}$ is defined by $\langle\varphi\rangle(\theta)=|\varphi(\theta)|_{E}$ for $\theta \in R^{-}$.

If all the hypotheses of Theorem 4.1 are satisfied except that the condition $\left(\Omega_{1}\right)$ is replaced by the condition $\left(\Omega_{4}\right)$, then the conclusion of Theorem 4.1 remains valid.

The following result is a modification of Theorems 4.1 and 4.2, which is very useful in studying delay differential equations (see Section 6).

THEOREM 4.4. Suppose that all the hypotheses of Theorem 4.1 are satisfied except that the conditions $\left(\Omega_{1}\right)$ and $\left(\mathrm{F}_{4}\right)$ are replaced by the condition $\left(\Omega_{2}\right)$ and the following condition:

$\left(\mathrm{F}_{\mathrm{b}}\right) \quad$ If $x, y \in X_{\sigma}^{\varphi}\left[t_{0}+d-\sigma\right], x_{t}, y_{t} \in S\left(\xi_{t_{0}}, r\right)$ and $|x-y|_{[\sigma, t]} \leqq 2 r$ for $t \in\left(t_{0}, t_{0}+d\right]$, then

$$
\left|f\left(t, x_{t}\right)-f\left(t, y_{t}\right)\right| \leqq \omega\left(t,|x-y|_{[\sigma, t]}\right)
$$

for a.a. $t \in\left(t_{0}, t_{0}+d\right]$.

Then the conclusion of Theorem 4.1 remains valid.

Remark 4.1. If $\omega$ is a Perron-type function, then the condition $\left(\Omega_{4}\right)$ in Theorem 4.3 can be replaced by the condition $\left(\Omega_{5}\right)$. Theorem 4.3 is a generalization of Theorems 4.1 and 4.2 under the additional axiom $\left(\mathrm{B}_{4}\right)$ and the condition (A). However, we showed in [9] that there exists an example for the phase space which does not satisfy the axiom $\left(\mathrm{B}_{4}\right)$. Theorem 4.4 is essentially a corollary to Theorem 4.1 (see [9]). So, the proof of Theorem 4.4 is omitted. We emphasize that both Theorems 4.3 
and 4.4 are useful in showing the convergence of successive approximations for (IP). For more detailed discussion see examples in Section 6 of the present paper.

Remark 4.2. In general, Theorem 4.4 is different from Theorem 4.1. In fact, it follows from $\left(\mathrm{F}_{4}\right)$ and $\left(\mathrm{B}_{2}\right)$ that

$$
\omega\left(t,\left|x_{t}-y_{t}\right|_{\mathscr{\sigma} R}\right) \leqq \omega\left(t, K(t-\sigma)|x-y|_{[\sigma, t]}\right)
$$

for $x, y \in X_{o}^{\varphi}\left[t_{0}+d-\sigma\right]$. The condition $\left(\Omega_{1}\right)$, however, is an assumption on the equation (2.1); it says nothing about the equation

$$
\frac{d z}{d t}=\omega(t, K(t-\sigma) z(t)) \text {. }
$$

Thus we cannot apply Theorem 4.4 to prove Theorem 4.1.

When $f$ in (1.1) is continuous, the following theorem holds.

THEOREM 4.5. Let $f$ be a continuous mapping from $J \times \mathscr{B}^{E}$ into $E$ and satisfy the condition $\left(\mathrm{F}_{3}\right)$. If the condition $\left(\Omega_{i}\right), i=1,3,4,2$, in Theorems 4.1-4.4 are replaced by the condition $\left(\Omega_{i}\right)$ with $\left(D^{+} z\right)\left(t_{0}\right)=0$, $i=1,3,4,2$, respectively, then the successive approximations $y^{n}(t)$ are well defined on $J$ and the sequence $\left\{y^{n}(t)\right\}$ converges uniformly on every compact interval of $J$ to a unique $C^{1}$-solution of (IP).

Finally, we shall give some sufficient conditions for the condition (i) in Theorem 4.1 .

(1) There exists a locally integrable function $\beta(t)$ such that

$$
|f(t, \psi)| \leqq \beta(t) \quad \text { for a.a. } t \in[\sigma, \infty) \text { and all } \psi \in \mathscr{B}^{E} \text {. }
$$

For a proof see [9, Lemma 5.1] (see also [7], [11]).

(2) There exist locally integrable functions $\rho(t)$ and $l(t)$ such that $|f(t, \psi)| \leqq \rho(t)|\psi|_{\mathscr{\infty}} E+l(t) \quad$ for a.a. $t \in[\sigma, \infty)$ and all $\psi \in \mathscr{B}^{E}$

(3) There exists a function $\omega(t, s)$ such that

$$
|f(t, \psi)| \leqq \omega\left(t,|\psi|_{\mathscr{B}}\right) \quad \text { for a.a. } t \in[\sigma, \infty) \text { and all } \psi \in \mathscr{B}^{E} \text {, }
$$

where $\omega(t, s)$ satisfies the following condition:

$$
|\omega(t, s)-\omega(t, \tau)| \leqq l(t)|s-t| \quad \text { for a.a. } t \in[\sigma, \infty) \text { and } s, \tau \in R^{+} \text {, }
$$

where $l(t)$ is locally integrable.

It is not difficult to prove (2) and (3).

REMARK 4.3. Our results apply to linear systems, though the results in [7], [11] do not. 
5. Proofs of theorems. Before proving the main theorems we present some lemmas.

LEMMA 5.1. $f_{n}:[a, b] \rightarrow E, n=1,2, \cdots$, be continuous, where $E$ is a Banach space. If the sequence $\left\{f_{n}(t)\right\}$ is equicontinuous on $[a, b]$ and convergent on a dense subset of $[a, b]$, then the sequence $\left\{f_{n}(t)\right\}$ converges uniformly on $[a, b]$.

For a proof of this lemma refer to Theorem 2.2 in [3].

LEMMA 5.2. Suppose that the conditions $\left(\mathrm{F}_{1}\right)-\left(\mathrm{F}_{3}\right)$ are satisfied. Then the following results on the successive approximations $y^{n}(t)$ are true.

(i) For each $y^{0} \in X_{o}^{\varphi}(a)$ the sequence $\left\{y^{n}(t)\right\}$ is well defined on $[\sigma, \sigma+a)$.

(ii) If the sequence $\left\{y^{n}(t)\right\}, y^{0} \in X_{\sigma}^{\varphi}(a)$, is uniformly bounded on $[\sigma, \gamma]$, $\gamma \in(\sigma, \sigma+a)$, then $\left\{y_{t}^{n}\right\}$ is uniformly bounded on $[\sigma, \gamma]$, and $\left\{y^{n}(t)\right\}$ and $\left\{y_{t}^{n}\right\}$ are equicontinuous on $[\sigma, \gamma]$.

For a proof of this lemma see [9, Lemma 5.1].

Proof OF THEOREM 4.1. Let $b=\sup \left\{\tau \geqq \sigma:\left\{y^{n}(t)\right\}\right.$ converges uniformly on $[\sigma, \tau]\}$. Then the inequality $b \geqq \sigma$ holds, since $y^{n}(\sigma)=\varphi(0)$. Assume $b<\sigma+a$; we show that this yields a contradiction. By Lemma 5.2 the sequence $\left\{y^{n}(t)\right\}$ is equicontinuous on $[\sigma, b]$ and by the definition of $b$ the sequence $\left\{y^{n}(t)\right\}$ converges to a continuous function uniformly on every compact interval of $[\sigma, b)$, provided $\sigma<b$. Thus the sequence $\left\{y^{n}(t)\right\}$ converges uniformly on $[\sigma, b]$ to a continuous function $y^{*}(t)$ by Lemma 5.1. If we set

$$
y(t)= \begin{cases}\varphi(t-\sigma) & \text { for } t \in(-\infty, \sigma] \\ y^{*}(t) & \text { for } t \in[\sigma, b],\end{cases}
$$

then $\left\{y_{b}^{n}\right\}$ converges to $y_{b} \in \mathscr{B}^{E}$, because of $\left(\mathrm{B}_{1}\right)$ and $\left(\mathrm{B}_{2}\right)$. By the assumption of this theorem, in correspondence with $(b, y) \in J \times X_{\sigma}^{\varphi}[b-\sigma]$, there exist positive constants $c, r$ and a Kamke-type function $\omega(t, s):(b, b+c] \times$ $[0,2 r] \rightarrow R^{+}$satisfying the condition $\left(\Omega_{1}\right)$ on $(b, b+c] \times[0,2 r]$ and the condition $\left(\mathrm{F}_{4}\right)$. Since $y_{b}^{n} \rightarrow y_{b}$ as $n \rightarrow \infty$, and since $\left\{y_{t}^{n}\right\}$ and $\left\{y^{n}(t)\right\}$ are equicontinuous on $[b, b+c]$ by Lemma 5.2 , there are $d, 0<d \leqq c$, and $N_{0}$ such that $\left\{y_{t}^{n} \in \mathscr{B}^{E}: t \in[b, b+d], n \geqq N_{0}\right\}$ is contained in $S\left(y_{b}, r\right)$ and that $\left|y^{m}-y^{n}\right|_{[\sigma, t]} \leqq 2 r$ for $t \in[b, b+d]$ and $m, n \geqq N_{0}$. Relabeling the numbers if necessary, we can assume $N_{0}=1$.

Next, we shall show that the sequence $\left\{y^{n}(t)\right\}$ converges uniformly on $[b, b+d]$. Put $\quad w^{m, n}(t)=\left|y^{m}(t)-y^{n}(t)\right|, \quad w^{k}(t)=\sup \left\{w^{m, n}(t): m, n \geqq k\right\}$, $z^{m, n}(t)=\left|y_{t}^{m}-y_{t}^{n}\right|$, and $z^{k}(t)=\sup \left\{z^{m, n}(t): m, n \geqq k\right\}$ for $t \in[b, b+d]$. Since 
$\left\{y^{n}(t)\right\}$ and $\left\{y_{t}^{n}\right\}$ are uniformly bounded and equicontinuous on $[b, b+d]$ by Lemma 5.2, $\left\{w^{k}(t)\right\}$ and $\left\{z^{k}(t)\right\}$ are also uniformly bounded and equicontinuous on $[b, b+d]$. Moreover, it is obvious that $0 \leqq w^{k}(t) \leqq w^{k-1}(t)$ and $0 \leqq z^{k}(t) \leqq z^{k-1}(t)$ for each $t \in[b, b+d], k=1,2, \cdots$, and hence, the sequences $\left\{w^{k}(t)\right\}$ and $\left\{z^{k}(t)\right\}$ converge pointwise on $[b, b+d]$. Therefore, by using Lemma 5.1 , we see that the sequences $\left\{w^{k}(t)\right\}$ and $\left\{z^{k}(t)\right\}$ converge uniformly to functions $w(t)$ and $z(t)$ defined on $[b, b+d]$, respectively.

The limit function $w(t)$ is an absolutely continuous function defined on $[b, b+d]$. Indeed, from $\left(\mathrm{F}_{3}\right)$ there exists a locally integrable function $\beta_{S}(t)$ defined on $J$, where $S=S\left(y_{b}, r\right)$. Then $H(t)=\int_{b}^{t} \beta_{S}(\tau) d \tau$ is an absolutely continuous function on $[b, b+d]$. From the definition of $w^{k}(t)$, we have

$$
\left|w^{k}(t)-w^{k}(s)\right| \leqq \sup _{m, n \geqq k}\left|w^{m, n}(t)-w^{m, n}(s)\right| \leqq 2|H(t)-H(s)|,
$$

which implies $|w(t)-w(s)| \leqq 2|H(t)-H(s)|$. Hence $w(t)$ is absolutely continuous on $[b, b+d]$.

From the condition $\left(\mathrm{F}_{4}\right)$ and the nondecreasing property of $\omega$, we have $\left|f\left(s, y_{s}^{m-1}\right)-f\left(s, y_{s}^{n-1}\right)\right| \leqq \omega\left(s,\left|y_{s}^{m-1}-y_{s}^{n-1}\right|\right) \leqq \omega\left(s, z^{k-1}(s)\right)$ for a.a. $s \in(b, b+d]$. The condition $\left(\omega_{2}\right)$ says that, if $[t, t+\Delta t] \subset(b, b+d)$, then $\omega\left(s, z^{k-1}(s)\right) \leqq$ $\alpha(s)$ for a.a. $s \in[t, t+\Delta t]$ and $\alpha(s)$ is integrable over $[t, t+\Delta t]$. Thus for $t \in(b, b+d)$ and for sufficiently small $\Delta t>0$ we have

$$
\begin{aligned}
\left|\Delta y^{m}(t)-\Delta y^{n}(t)\right| & \leqq \int_{t}^{t+\measuredangle t}\left|f\left(s, y_{s}^{m-1}\right)-f\left(s, y_{s}^{n-1}\right)\right| d s \\
& \leqq \int_{t}^{t+\measuredangle t} \omega\left(s, z^{k-1}(s)\right) d s \quad \text { for } m, n \geqq k,
\end{aligned}
$$

where $\Delta y^{i}(t)=y^{i}(t+\Delta t)-y^{i}(t), i=m, n$. Therefore

$$
\left|w^{k}(t+\Delta t)-w^{k}(t)\right| \leqq \int_{t}^{t+\measuredangle t} \omega\left(s, z^{k-1}(s)\right) d s .
$$

Letting $k \rightarrow \infty$, we obtain, by the Lebesgue dominated convergence theorem,

$$
|w(t+\Delta t)-w(t)| \leqq \int_{t}^{t+\Delta t} \omega(s, z(s)) d s .
$$

Dividing both sides by $\Delta t>0$ and letting $\Delta t \rightarrow 0$, we have

$$
\left|\frac{d w}{d t}\right| \leqq \omega(t, z(t)) \quad \text { for a.a. } t \in(b, b+d) .
$$

On the other hand, we have by $\left(B_{2}\right)$,

$$
z^{m, n}(t) \leqq K(t-b) \sup \left\{w^{m, n}(s): b \leqq s \leqq t\right\}+M(t-b) z^{m, n}(b),
$$


so that

$$
z^{k}(t) \leqq K(t-b) \sup \left\{w^{k}(s): b \leqq s \leqq t\right\}+M(t-b) z^{k}(b) .
$$

Letting $k \rightarrow \infty$ in the above inequality, we obtain

$$
z(t) \leqq K(t-b) \sup \{w(s): b \leqq s \leqq t\} .
$$

Thus, it follows from (5.4), (5.5) and $\left(\omega_{1}\right)$ that

$$
\left|\frac{d w}{d t}\right| \leqq \omega\left(t, K(t-b) \sup _{b \leqq s \leqq t} w(s)\right) \text {. }
$$

Since $w(b)=0$, we see that $w(t) \equiv 0$ on $[b, b+d]$ by Lemma 3.4. This implies that the sequence $\left\{y^{n}(t)\right\}$ converges uniformly on $[b, b+d]$, which contradicts the definition of $b$. Thus we have $b=\sigma+a$.

Since the sequence $\left\{y^{n}(t)\right\}$ converges uniformly to a function $y^{*}(t)$ on every compact interval of $J$, a function $y(t)$ is defined on $(-\infty, \sigma+b)$ in the same way as in (5.1). Then, from the dominated convergence theorem for vector-valued functions, it follows that

$$
\lim _{n \rightarrow \infty} \int_{\sigma}^{t} f\left(s, y_{s}^{n}\right) d s=\int_{\sigma}^{t} f\left(s, y_{s}\right) d s \quad \text { for each } t \in[\sigma, \sigma+a) .
$$

This implies that

$$
y(t)= \begin{cases}\varphi(t-\sigma) & \text { for } t \in(-\infty, \sigma] \\ \varphi(0)+\int_{\sigma}^{t} f\left(s, y_{s}\right) d s & \text { for } t \in[\sigma, \sigma+a) .\end{cases}
$$

Thus the function $y(t)$ is a solution of (IP).

Finally, we shall show that $y(t)$ is the unique solution of (IP) on $J$. Let $x(t)$ be another solution of (IP) on $[\sigma, \sigma+h), 0<h \leqq a$. Suppose $\sigma \leqq \zeta \leqq \sigma+h$, where $\zeta=\sup \{t \geqq \sigma: x(s)=y(s)$ for $s \in(-\infty, t]\}$. From the assumption of the theorem, in correspondence with $\left(\zeta, y_{\zeta}\right) \in J \times \mathscr{B}^{E}$ there exist positive constants $h_{1}, 0<h_{1}<h, r$ and a Kamke-type function $\omega:\left(\zeta, \zeta+h_{1}\right] \times[0,2 r] \rightarrow R^{+}$satisfying the condition $\left(\Omega_{1}\right)$ and the condition $\left(\mathrm{F}_{4}\right)$. If we set $u(t)=|x(t)-y(t)|$ for $t \in\left(-\infty, \zeta+h_{1}\right]$ then we have

$$
\left|\frac{d u}{d t}\right| \leqq\left|\frac{d x}{d t}-\frac{d y}{d t}\right|=\left|f\left(t, x_{t}\right)-f\left(t, y_{t}\right)\right| \leqq \omega\left(t,\left|x_{t}-y_{t}\right|_{\mathscr{B}} E\right),
$$

and hence $|d u / d t| \leqq \omega(t, K(t-\zeta) \sup \{u(s): \zeta \leqq s \leqq t\})$ as long as $\left|x_{s}-y_{\zeta}\right|<r$, $\left|y_{s}-y_{\zeta}\right|<r$ and $u(s) \leqq 2 r / K_{h_{1}}$ for $s \in[\zeta, t]$, where $\zeta<t \leqq \zeta+h_{2}$ for some $h_{2} \leqq h_{1}$. Thus it follows from Lemma 3.4 that $u(t) \equiv 0$ on $\left[\zeta, \zeta+h_{2}\right]$, and hence, $u(t) \equiv 0$ on $J$.

q.e.d.

Since the argument for the proof of the theorems below is similar 
to that in Theorem 4.1, we shall only sketch the parts that need modifications by using the same notation as in the proof of Theorem 4.1.

Proof of THEOREM 4.2. We shall consider the estimate for $z^{m, n}(t+\Delta t)$ instead of the difference $\left|y^{m}(t)-y^{n}(t)\right|$ in (5.2). For $t \in$ $(b, b+d)$ and for sufficient small $\Delta t>0$ we have

$$
\begin{aligned}
z^{m, n}(t+\Delta t) & \leqq\left|y_{t+\Delta t}^{m}-S(\Delta t) y_{t}^{m}+S(\Delta t) y_{t}^{n}-y_{t+\Delta t}^{n}\right|+\|S(\Delta t)\| z^{m, n}(t) \\
& \leqq K(\Delta t) \sup _{t \leq s \leq t+L t}\left|y^{m}(s)-y^{m}(t)+y^{n}(t)-y^{n}(s)\right|+\|S(\Delta t)\| z^{m, n}(t) \\
& \leqq K(\Delta t) \int_{t}^{t+\Delta t} \omega\left(s, z^{m-1, n-1}(s)\right) d s+\|S(\Delta t)\| z^{m, n}(t),
\end{aligned}
$$

and hence, we have

$$
z^{k}(t+\Delta t) \leqq K(\Delta t) \int_{t}^{t+\Delta t} \omega\left(s, z^{k-1}(s)\right) d s+\|S(\Delta t)\| z^{k}(t),
$$

that is,

$$
z^{k}(t+\Delta t)-z^{k}(t) \leqq K(\Delta t) \int_{t}^{t+\Delta t} \omega\left(s, z^{k-1}(s)\right) d s+\{\|S(\Delta t)\|-1\} z^{k}(t) .
$$

Since $z^{k}(t) \rightarrow z(t)$ as $k \rightarrow \infty$, we have

$$
z(t+\Delta t)-z(t) \leqq K(\Delta t) \int_{t}^{t+\Delta t} \omega(s, z(s)) d s+\{\|S(\Delta t)\|-1\} z(t) .
$$

Dividing both sides by $\Delta t$ and letting $\Delta t \rightarrow 0+$, we obtain

$$
\left(\bar{D}_{+} z\right)(t) \leqq K(0) \omega(t, z(t))+\beta_{2} z(t),
$$

because of (2.4) and $\left(\mathrm{B}_{2}\right)$. Moreover, $z(t)$ is absolutely continuous on $[b, b+d]$ since $w(t) \equiv 0$ on $(-\infty, b]$ and $w(t)$ is absolutely continuous on $[b, b+d]$ (see [10, Lemma 3.3]). Thus we have

$$
\frac{d z}{d t} \leqq K(0) \omega(t, z(t))+\beta_{\llcorner} z(t) \quad \text { for a.a. } t \in(b, b+d] .
$$

By using Lemma 3.5 , we can see that $z(t) \equiv 0$ on $[b, b+d]$. This contradicts the definition of $b$.

Next, setting $v(t)=x(t)-y(t)$, we have, by (5.7) and Lemma 3.1,

$$
\bar{D}_{+}\left|v_{t}\right| \leqq K(0)\left|\frac{d v}{d t}\right|+\beta_{\nu}\left|v_{t}\right| \leqq K(0) \omega\left(t,\left|v_{t}\right|\right)+\beta_{\nu}\left|v_{t}\right| \cdot
$$

Thus it follows from Lemma 3.5 that $\left|v_{t}\right| \equiv 0$ on $\left[\xi, \xi+h_{2}\right]$, and so $v(t) \equiv 0$ on $\left[\xi, \xi+h_{2}\right]$ by $\left(\mathrm{B}_{3}\right)$. This implies that (IP) has a unique solution, which completes the proof of Theorem 4.2.

q.e.d.

Proof OF THEOREM 4.3. Put $\widetilde{z}^{m, n}(t)=\left|w_{t}^{m, n}\right|$ and $\widetilde{z}^{k}(t)=\sup \left\{\widetilde{z}^{m, n}(t)\right.$ : 
$m, n \geqq k\}$ for all $t \in[b, b+d]$, where $w^{m, n}(t)=\left|y^{m}(t)-y^{n}(t)\right|$ for all $t \in$ $(-\infty, b+d]$. By $\left(\mathrm{B}_{1}\right),\left(\mathrm{B}_{4}\right)$ and $(\mathrm{A})$, it is not difficult to show that the relation

$$
z^{k}(t) \leqq \widetilde{z}^{k}(t) \leqq\left|w_{i}^{k}\right| \text { on } \quad[b, b+d]
$$

holds. Further, since $\left\{w^{k}(t)\right\}$ converges to a function $w(t)$ uniformly on $(-\infty, b+d]$, it follows that $\left\{\left|w_{t}^{k}\right|\right\}$ converges to a function $\left|w_{t}\right|$ for every $t \in[b, b+d]$. Thus by (5.2) and (5.8) we have

$$
\left|w^{k}(t+\Delta t)-w^{k}(t)\right| \leqq \int_{t}^{t+\Delta t} \omega\left(s, z^{k-1}(s)\right) d s \leqq \int_{t}^{t+\Delta t} \omega\left(s,\left|w_{s}^{k-1}\right|\right) d s
$$

as long as $\left|w_{s}^{k-1}\right| \leqq 2 r$ for all $s \in[b, b+d]$. Consequently, it follows that

$$
\left|\frac{d w}{d t}\right| \leqq \omega\left(t,\left|w_{t}\right|\right) \quad \text { for a.a. } t \in(b, b+c) .
$$

Hence $w(t) \equiv 0$ on $[b, b+d]$ by $\left(\Omega_{4}\right)$.

Next, by (5.7), we have $|d u / d t| \leqq \omega\left(t,\left|x_{t}-y_{t}\right|\right) \leqq \omega\left(t,\left|\langle x-y\rangle_{t}\right|\right) \leqq$ $\omega\left(t,\left|u_{t}\right|_{\circledast} R\right)$. Thus, by $\left(\Omega_{4}\right)$, (IP) has a unique solution. q.e.d.

Proof of Theorem 4.5. We shall only show that $\left(D^{+} w\right)(b)=0$ and $\left(D^{+} z\right)(b)=0$. By the continuity of $f$, for any $\varepsilon>0$, there exist positive numbers $\delta$ and $\eta$ such that $\left|f(t, \psi)-f\left(b, y_{b}\right)\right|<\varepsilon / 2$ for all $(t, \psi) \epsilon$ $[b, b+\delta] \times S\left(y_{b}, \eta\right)$, where $0<\delta \leqq d$ and $0<\eta \leqq r$. Since the sequence $\left\{y_{t}^{n}\right\}$ is equicontinuous on $[b, b+\delta]$ and $y_{b}^{n} \rightarrow y_{b}$ as $n \rightarrow \infty$, there exist a positive number $l, 0<l \leqq \delta$, and an integer $N_{1} \geqq N_{0}$ such that $\left\{y_{t}^{n} \in \mathscr{B}^{E}\right.$ : $\left.n \geqq N_{1}\right\} \subset S\left(y_{b}, \eta\right)$ for all $t \in[b, b+l]$. Thus, we have,

$$
w^{k}(t) \leqq \sup _{m, n \geqq k}\left\{\int_{b}^{t}\left|f\left(s, y_{s}^{m-1}\right)-f\left(b, y_{b}\right)\right| d s+\int_{b}^{t}\left|f\left(s, y_{s}^{n-1}\right)-f\left(b, y_{b}\right)\right| d s\right\}+w^{k}(b),
$$

which implies $w^{k}(t) \leqq \varepsilon(t-b)+w^{k}(b)$, and hence, letting $k \rightarrow \infty$, we get $w(t) \leqq \varepsilon(t-b)$. This implies $\left(D^{+} w\right)(b)=0$.

Moreover, in view of (5.5) and the above result, we have $z(t) \leqq$ $\varepsilon(t-b) K(t-b)$, which implies that $\left(D^{+} z\right)(b)=0 . \quad$ q.e.d.

6. Examples. In this section, the results on the uniqueness of solutions and the global convergence of successive approximations obtained in Section 4 are illustrated in examples. In particular, we note that Examples 6.1 and 6.2 stated below imply that, in general, both Theorems 4.3 and 4.4 are useful in showing the uniqueness and the convergence of successive approximations of solutions for (IP), provided the conditions $\left(\Omega_{i}\right), i=2,4$, are replaced by the conditions $\left(\Omega_{i}\right)$ with $\left(D^{+} z\right)\left(t_{0}\right)=0$. It is easy to see that the following result holds (cf. [4]). 
Proposition 6.1. Let $\varepsilon(t)$ be a positive and continuous function defined on $\left(t_{0}, t_{0}+d\right]$ such that

$$
\int_{t_{0}}^{t_{0}+d} \frac{\varepsilon(t)}{t-t_{0}} d t<\infty
$$

Then the initial value problem

$$
\begin{aligned}
& \frac{d z}{d t}=\frac{1+\varepsilon(t)}{t-t_{0}} z(t) \\
& \lim _{t \rightarrow t_{0}+0} z(t)=\left(D^{+} z\right)\left(t_{0}\right)=0
\end{aligned}
$$

has only zero solution.

EXAMPLE 6.1. We consider the scalar functional differential equation

$$
\begin{aligned}
& \frac{d x}{d t}=\frac{3}{2}(t-\sigma-2) \sin \frac{x((t+\sigma) / 2)}{(t-\sigma-2)^{2}}, \\
& x_{\sigma}=\varphi \in \mathscr{C}_{r}^{R},
\end{aligned}
$$

where the phase space $\mathscr{C}_{r}^{R}, \gamma=\log (2 / 3)<0$, is as in Example 2.1 with $R$ as $E$. Let $f(t, \psi):[\sigma, \infty) \times \mathscr{C}_{r}^{R} \rightarrow R$ be defined as follows:

$$
f(t, \psi)=\frac{3}{2}(t-\sigma-2) \sin \frac{\psi((\sigma-t) / 2)}{(t-\sigma-2)^{2}},
$$

Clearly, $f(t, \psi)$ is continuous in $(t, \psi) \in[\sigma, \infty) \times \mathscr{C}_{r}^{R}$ and $|f(t, \psi)| \leqq$ $3|t-\sigma-2| / 2$. It is sufficient to examine the conditions in the theorems when $t_{0}=\sigma+2$. Corresponding to $\left(\mathrm{F}_{4}\right)$ and $\left(\mathrm{F}_{5}\right)$, we have

$$
\begin{aligned}
\left|f\left(t, x_{t}\right)-f\left(t, y_{t}\right)\right| & \leqq \frac{3}{2}\left|x_{t}\left(\frac{\sigma-t}{2}\right)-y_{t}\left(\frac{\sigma-t}{2}\right)\right| /(t-\sigma-2) \\
& \leqq \frac{3}{2} e^{-\gamma(\sigma-t) / 2}\left|x_{t}-y_{t}\right|_{\mathscr{E}_{r}^{R}}^{R} /(t-\sigma-2) \\
& \leqq \frac{3}{2} e^{r}\left|x_{t}-y_{t}\right|_{\mathscr{F}_{r}^{R}}^{R /(t-\sigma-2)} \\
& \leqq\left|x_{t}-y_{t}\right|_{\sigma_{r}^{R}}^{R} /(t-\sigma-2)
\end{aligned}
$$

and

$$
\left|f\left(t, x_{t}\right)-f\left(t, y_{t}\right)\right| \leqq \frac{3}{2}|x-y|_{[\sigma, t]} /(t-\sigma-2),
$$

respectively. Then (2.1), (2.5), (2.6) and (2.2) become

$$
\frac{d z}{d t}=e^{\gamma(\sigma+2-t)} z(t) /(t-\sigma-2), \quad\left(K(t)=e^{-\gamma t} \text { in }\left(\mathrm{B}_{2}\right)\right)
$$




$$
\begin{aligned}
& \frac{d z}{d t}=\frac{z(t)}{t-\sigma-2}-\gamma z(t), \quad\left(\beta_{\nu}=-\gamma\right) \\
& \left|\frac{d z}{d t}\right| \leqq\left|z_{t}\right|_{\gamma}^{R} /(t-\sigma-2)
\end{aligned}
$$

and

$$
\frac{d z}{d t}=\frac{3}{2} z(t) /(t-\sigma-2),
$$

respectively. Therefore it follows from (6.3) (or (6.2)) that the condition $\left(\Omega_{3}\right)$ with $\left(D^{+} z\right)(\sigma+2)=0$ is satisfied, and hence the conclusion of Theorem 4.2 for IP(6.1) holds. However, from (6.4) the condition $\left(\Omega_{2}\right)$ with $\left(D^{+} z\right)(\sigma+2)=0$ is not satisfied.

EXAMPLE 6.2. We consider the scalar integro-differential equation

$$
\begin{aligned}
& \frac{d x}{d t}=(t-\sigma)\left\{\sin \frac{x(t)}{(t-\sigma)^{2}}+\left(1+e^{-(t-\sigma)}\right) \int_{-\infty}^{t} e^{-(t-s)} \sin \frac{x(s)}{(t-\sigma)^{2}} d s\right\}, \\
& x_{\sigma}=\varphi \in \mathscr{L}^{R},
\end{aligned}
$$

where $\mathscr{L}^{R}$ is as in Example 2.2 with $r=0$. Let $f(t, \psi):[\sigma, \infty) \times$ $\mathscr{L}^{R} \rightarrow R$ be defined as

$$
f(t, \psi)=(t-\sigma)\left\{\sin \frac{\psi(0)}{(t-\sigma)^{2}}+\left(1+e^{-(t-\sigma)}\right) \int_{-\infty}^{0} e^{\theta} \sin \frac{\psi(\theta)}{(t-\sigma)^{2}} d \theta\right\} .
$$

Evidently, $f(t, \psi)$ is continuous in $(t, \psi) \in[\sigma, \infty) \times \mathscr{L}^{R}$ and $|f(t, \psi)| \leqq$ $3|t-\sigma|$. Corresponding to $\left(\mathrm{F}_{4}\right)$ and $\left(\mathrm{F}_{5}\right)$ with $t_{0}=\sigma$, we have

$$
\begin{aligned}
\mid f(t, & \left.x_{t}\right)-f\left(t, y_{t}\right) \mid \\
& \leqq\left\{|x(t)-y(t)|+\left(1+e^{-(t-\sigma)}\right) \int_{-(t-\sigma)}^{0} e^{\theta}|x(t+\theta)-y(t+\theta)| d \theta\right\} /(t-\sigma) \\
& \leqq \frac{1+e^{-(t-\sigma)}}{t-\sigma}\left|x_{t}-y_{t}\right|_{\mathscr{E} R}
\end{aligned}
$$

and

$$
\left|f\left(t, x_{t}\right)-f\left(t, y_{t}\right)\right| \leqq \frac{2-e^{-2(t-o)}}{t-\sigma}|x-y|_{[\sigma, t]},
$$

respectively. Then (2.1), (2.5), (2.6) and (2.2) become

$$
\begin{gathered}
\frac{d z}{d t}=\frac{2+e^{-(t-\sigma)}-e^{-2(t-\sigma)}}{t-\sigma} z(t), \quad\left(K(t)=2-e^{-t} \text { in }\left(\mathrm{B}_{2}\right)\right), \\
\frac{d z}{d t}=\frac{1+e^{-(t-\sigma)}}{t-\sigma} z(t)+\beta_{\nu} z(t), \quad\left(0 \leqq \beta_{\nu} \leqq 1\right)
\end{gathered}
$$




$$
\left|\frac{d z}{d t}\right| \leqq \frac{1+e^{-(t-\sigma)}}{t-\sigma}\left|z_{t}\right|_{\mathscr{x}}
$$

and

$$
\frac{d z}{d t}=\frac{2-e^{-2(t-\sigma)}}{t-\sigma} z(t)
$$

respectively. Therefore it follows from (6.8) that the condition $\left(\Omega_{2}\right)$ with $\left(D^{+} z\right)(\sigma)=0$ is satisfied, and hence the conclusion of Theorem 4.4 for IP (6.5) holds. However, from (6.7) the condition $\left(\Omega_{3}\right)$ with $\left(D^{+} z\right)(\sigma)=0$ is not satisfied.

\section{REFERENCES}

[1] E. Coddington and N. Levinson, Theory of Ordinary Differential Equations, McGrawHill, New York, 1955.

[2] J. K. Hale and J. Kato, Phase space for retarded equations with infinite delay, Funkcial. Ekvac. 21 (1978), 11-41.

[3] P. Hartman, Ordinary Differential Equations, John Wiley and Sons, New York, 1964.

[4] M. Hukuhara, Ordinary Differential Equations (in Japanese), Iwanami-Shoten, Tokyo, 1980.

[5] V. Lakshmikantham and S. Leela, Differential and Integral Inequalities, Vol. I, II, Academic Press, New York, 1969.

[6] V. Lakshmikantham and S. Leela, Nonlinear Differential Equations in Abstract Spaces, Pergamon Press, Oxford, New York, 1981.

[7] S. KATO, On the convergenece of successive approximations for nonlinear ordinary differential equations in a Banach space, Funkcial. Ekvac. 21 (1978), 43-52.

[8] K. SchUMACHER, Existence and continuous dependence for functional differential equations with infinite delay, Arch. Rational Mech. Anal. 7 (1978), 315-334.

[9] J.S. SHIN, Uniqueness and global convergence of successive approximations for solutions of functional integral equations with infinite delay, J. Math. Anal. Appl. 120 (1986), $71-88$.

[10] J.S. SHIN, On the uniqueness of solutions for functional differential equations with infinite delay, to appear in Funkcial. Ekvac.

[11] G. Vidossich, Global convergence of successive approximations, J. Math. Anal. Appl. 45 (1974), 285-292.

Department of Mathematics

KOREA UNIVERSITY

KODAIRA, TOKYO 187

JAPAN 